\title{
New reconstruction of the event-integrated spectra for GLE events
}

\section{Sergey Koldobskiy, ${ }^{a, b, *}$ Osku Raukunen, ${ }^{c}$ Rami Vainio, ${ }^{c}$ Gennady Kovaltsov ${ }^{d}$ and llya Usoskin ${ }^{a}$}

${ }^{a}$ University of Oulu, Finland

${ }^{b}$ National Research Nuclear University MEPhI, Moscow, Russia

${ }^{c}$ University of Turku, Finland

${ }^{d}$ Ioffe Physical-Technical Institute, St. Petersburg, Russia

E-mail: sergey.koldobskiy@oulu.fi

Here we report a new reconstruction of the event-integrated spectra of solar energetic particles (SEP) detected by neutron monitor (NM) network and space-borne detectors (mainly GOES data) during ground-level enhancement (GLE) events. The reconstruction of SEP fluences is based on the "bow-tie" method employing the latest advances in the NM data analysis (a timedependent GCR background and the use of the NM yield function directly verified with the AMS-02 experiment data), usage of different space-borne detectors data and a detailed study of different uncertainties. As a result of this work, we obtained integral fluences of SEPs in the energy range from $30 \mathrm{MeV}$ to a few $\mathrm{GeV}$ for 58 moderate and strong GLE events since 1956. The results were fitted with the modified Band-function (a double power-law function with two exponential cutoffs). An easy-to-use presentation of SEP fluences in the form of an analytical expression forms a solid basis for new studies in different fields, such as the influence of SEPs on the atmosphere and a statistical study of extreme solar activity.

$37^{\text {th }}$ International Cosmic Ray Conference (ICRC 2021)

July 12th - 23rd, 2021

Online - Berlin, Germany

\footnotetext{
*Presenter
} 


\section{Introduction}

Fluxes of charged particles, called cosmic rays (CRs), permanently bombard Earth. They mostly originate either from Galactic and extra-galactic sources. From the 1970s, spectra of cosmic rays were directly observed by different satellite-based cosmic-ray experiments. CRs having high enough energy ( $\geq \sim 0.8 \mathrm{GeV}$ ) can penetrate the Earth's magnetosphere and initiate hadron-lepton cascades of secondary particles. These cascades can be registered by different ground-based detectors, including neutron monitors (NM), which are suitable for the detection of hadronic component of secondary particles.

Sporadically during solar eruptive events, such as solar flares and coronal mass ejections, charged particles can be accelerated up to several $\mathrm{GeV}$ energy, these particles are known as solar energetic particles (SEPs). SEP events which can be registered by ground-based detectors are called ground level enhancements (GLEs) [1]. Up to date we know 72 GLE events, numbered incrementally from GLE \#1 occurred on 28 February 1942. First four ones were registered with ionization chambers [2] and currently unavailable for a quantitative analysis. But GLEs starting from GLE \#05 (23-Feb-1956) were recorded by the worldwide NM network and are currently publicly available for analyses using the International GLE Database (IGLED, https://gle . oulu . fi).

The analysis of GLEs plays a crucial role in our understanding of processes of SEP acceleration and propagation and related topics (SEP radiation hazard, the study of extreme SEP events using cosmogenic isotopes, etc.). Depending on the analysis method, different SEP characteristics can be revealed. In this study, we focus on the reconstruction of integral fluences for the most powerful SEP events in the NM era (starting from the 1950s up today). This kind of reconstruction requires a simultaneous analysis of ground-based and satellite-based data and was performed previously [3,4] but here we present updated analysis. First, a significantly updated NM yield function $[5,6]$ directly verified [7] with AMS-02 experiment data [8] was used. Second, for NM data we used a recently developed method of the effective NM energy [9] (similar to the "bow-tie" method [10]) which was verified [11] using PAMELA experimental data [12]. Third, GLE NM responses corrected for variable galactic cosmic ray (GCR) flux were used [13], that is particularly important for an analysis of events that occurred during the recovery phase of Forbush decreases. Fourth, carefully reconstructed GOES data [14] were used in the low-energy region. All these features make the new reconstruction the most accurate one.

Here we shortly describe the key elements of analysis performed in $[9,11,13,15]$, please refer to the original papers for details of the method and parametrization of obtained fluences.

\section{Data collection}

\subsection{NM data}

Here we used a recent reconstruction of high-energy $(E>800 \mathrm{MeV})$ integral fluxes of SEPs [13]. The main advantages of this analysis are discussed above. The reconstruction of integral fluences was performed for 58 moderate and strong GLE events. Several weak ones were discarded due to the impossibility of clear reconstruction of SEP signal on GCR background. 


\subsection{Satellite and ionospheric data}

For GLEs before \#40 we used all available data, including satellite and ionospheric reconstructions [16-20]. For GLEs starting from \#40 we used the integral channels $>30 \mathrm{MeV},>50$ $\mathrm{MeV},>60 \mathrm{MeV}$ and $>100 \mathrm{MeV}$ from GOES/EPS and GOES/EPEAD as well as higher energy "bow-tie"-calculated integral channels from GOES/HEPAD [14]. For GLE \#71 (17-May-2012) we also included PAMELA experiment data [12] into the analysis.

\section{Parametrization of fluxes}

Combined analysis of NM and satellite/ionospheric data allowed us to estimate fluences in a wide energy range from $\sim 30 \mathrm{MeV}$ to several $\mathrm{GeV}$. Reconstructed fluences were fitted with the modified Band function:

$$
\begin{aligned}
& F(>R)=J_{1}\left(\frac{R}{1 \mathrm{GV}}\right)^{-\gamma_{1}} \exp \left(-\frac{R}{R_{1}}\right) \text { if } R<R_{b} \\
& F(>R)=J_{2}\left(\frac{R}{1 \mathrm{GV}}\right)^{-\gamma_{2}} \exp \left(-\frac{R}{R_{2}}\right) \text { if } R \geq R_{b}
\end{aligned}
$$

where $F(>R)$ is the omnidirectional fluence (in units of $\mathrm{cm}^{-2}$ ) of particles with rigidity greater than $R$ (which is expressed in gigavolts), parameters $\gamma_{1}, \gamma_{2}, R_{1}, R_{2}$, and $J_{2}$ are defined by fitting, and other parameters can be calculated as

$$
\begin{aligned}
R_{b} & =\gamma_{0} \cdot R_{0} \\
J_{1} & =J_{2} \cdot R_{b}^{-\gamma_{0}} \cdot \exp \left(\gamma_{0}\right) \\
\gamma_{0} & =\gamma_{2}-\gamma_{1} \\
R_{0} & =R_{1} \cdot R_{2} /\left(R_{2}-R_{1}\right) .
\end{aligned}
$$

The main features of this function are that (i) it can make the smooth junction between low-energy and high-energy parts (including exponential cutoffs) and (ii) its first derivative, the differential flux of particles, is also smooth and can be analytically calculated from Eqs. 1, 2.

The fitting procedure was performed in three main steps.

First, the high-energy (NM) part of the fluence was fitted using Eq. 2. Both experimental points and upper limits were used in the analysis. Fitting was done using Monte-Carlo techniques, at each iteration we simulated a set of randomised points in coordinates $[F(>R), R]$ using calculated values [21] and also considering both statistic and systematic uncertainties. Moreover, in order to reduce the statistical weight of a more numerous polar/high-latitude NMs in the subsequent fitting procedure, the uniform Monte-Carlo selection of NM based on the cutoff rigidity value was applied, so that at each iteration only one NM inside the $0.4 \mathrm{GV}$ bin was selected. Obtained set of points was fitted with Eq. 2 applying a non-linear least-squares method and the best-fit parameters $J_{2}, \gamma_{2}$, and $R_{2}$ were found based on minimisation of the logarithmic residual.

Second, low-energy points were added in the analysis in a way similar to NM points. Parameters $J_{2}, \gamma_{2}$, and $R_{2}$ obtained from the NM points fit were fixed and parameters $\gamma_{1}$ and $R_{1}$ were found applying a non-linear least-squares method. 
Steps 1 and 2 were performed 5000 times and the best-fit values $\gamma_{1}, R_{1}, J_{2}, \gamma_{2}$, and $R_{2}$ were derived on the basis of the formal $\chi^{2}$ value calculation.

In the third step, an additional Monte-Carlo simulation was performed in order to obtain more precise values of parameters and to evaluate the uncertainty of derived best-fit parameters. On this step, parameters were varied using the normal distribution for each iteration and formal $\chi^{2}$ value for each iteration was computed. If the newly computed $\chi^{2}$ value was lower than that derived in Step 2 or previous iterations of Step 3, the corresponding set of parameters was chosen as a new best-fit and the procedure was restarted. 10000 iteration having the $\chi^{2}<\chi_{\min }^{2}+5.89$ (that corresponds to $68 \%$ confidence interval (c.i.) for a 5-parameter model) were used in this step, all the parameter values were recorded in order to evaluate the uncertainty of parameters (see Figure 1).

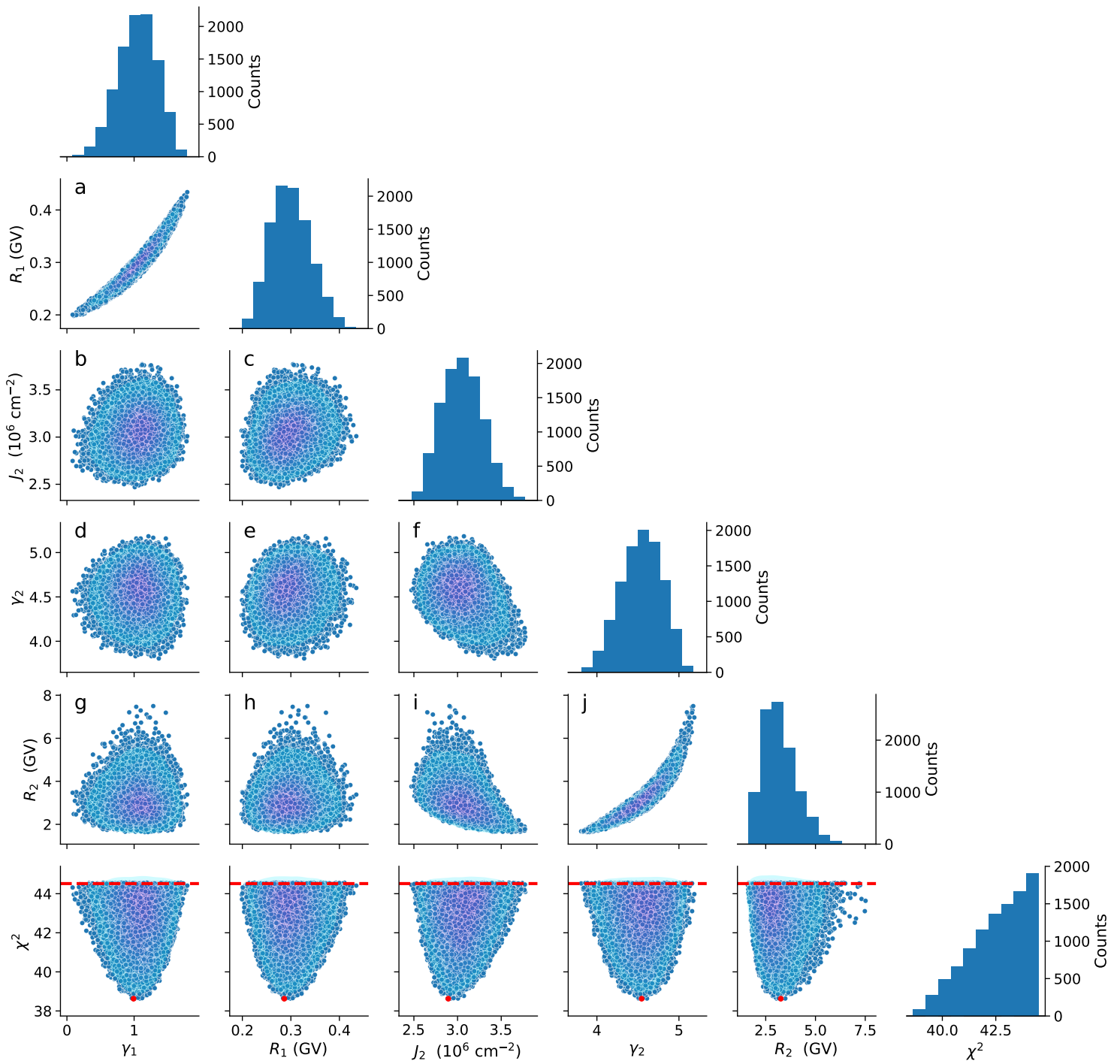

Figure 1: Distribution of obtained parameters and its pair-wise correlations $(a-j)$ together with $\chi^{2}$ vs. parameters distributions (bottom plots) for GLE \#60 after 10000 iterations of Step 3 (see discussion in the text). Red dots on $\chi^{2}$ vs parameters plots correspond to the best-fit ( $\chi_{\min }^{2}$ value) and the red dashed line corresponds to $68 \%$ confidence interval. The intensity of color corresponds to the point density. 


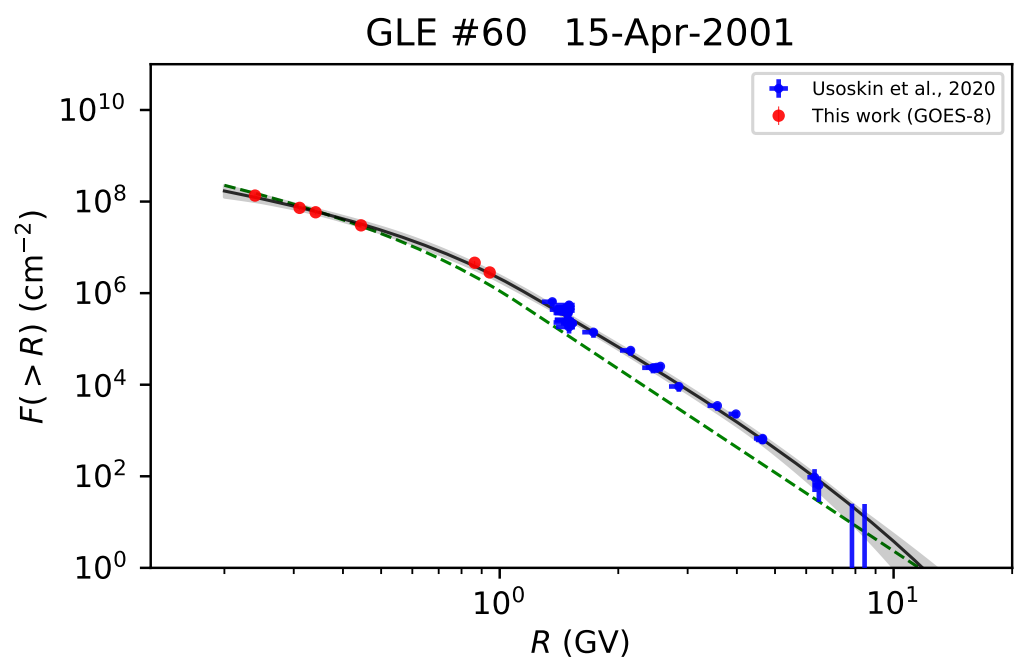

Figure 2: Reconstructed integral fluence for GLE \#60: red and blue points are results of satellite and NM data analysis, correspondingly. The black line is a best-fit, grey region across the black line is $68 \%$ confidence interval. The green dashed line is a fluence reconstruction from [4].

All three steps were applied to each of 58 analysed evens. Parameter values (together with uncertainties) and overall quality of the fit (calculated as a averaged ratio between higher and lower values of the fluence inside the $68 \%$ c.i.) for reconstructed fluences were obtained [15]. In Figure 2 the example of the obtained SEP fluence is given. The previous reconstruction (green dashed line in Fig. 2) of the SEP fluence [4] is 2-3 times lower in the high-energy NM region and does not have high-energy exponential cutoff, for most part of analysed GLE events the same effect is observed. Moreover, in some cases low-energy fluences are also significantly changed.

\section{Conclusion}

In this work we made a reconstruction of integral fluxes (fluences) for 58 most powerful SEP events during the last 70 years. The reconstruction is based on satellite/ionospheric and NM data, that were analysed using updated models and data analysis techniques. Provided parametrization of integral fluxes in the form of analytical functions and opportunity to perform the calculation of differential fluxes allows easy use of obtained results in different applications (ionization of atmosphere, production of cosmogenic isotopes, assessment of radiation doses).

\section{Acknowledgments}

Detrended data of GLE recorded by NMs were obtained from the International GLE database https://gle.oulu.fi. PIs and teams of all the ground-based neutron monitors and space-borne experiments whose data were used here, are gratefully acknowledged. This work was partially supported by the Academy of Finland (projects No. 321882 ESPERA, 330063 QUASARE). S.K. acknowledges support by the Russian Science Foundation project no. 20-72-10170. The work in the University of Turku was performed in the framework of the Finnish Centre of Excellence 
in Research of Sustainable Space funded by the Academy of Finland (grant no. 312357). The authors benefited from discussions within the ISSI International Team work (HERIOC team) and ISWAT-COSPAR S1-02 team.

\section{References}

[1] S.V. Poluianov, I.G. Usoskin, A.L. Mishev, M.A. Shea and D.F. Smart, GLE and Sub-GLE Redefinition in the Light of High-Altitude Polar Neutron Monitors, Solar Physics 292 (2017) 176 [1711.06161].

[2] S.E. Forbush, Three Unusual Cosmic-Ray Increases Possibly Due to Charged Particles from the Sun, Physical Review 70 (1946) 771.

[3] A. Tylka and W. Dietrich, A new and comprehensive analysis of proton spectra in ground-level enhanced (GLE) solar particle events, Proceedings of the 31st ICRC, Lodz (2009) 273.

[4] O. Raukunen, R. Vainio, A.J. Tylka, W.F. Dietrich, P. Jiggens, D. Heynderickx et al., Two solar proton fluence models based on ground level enhancement observations, Journal of Space Weather and Space Climate 8 (2018) A04.

[5] A.L. Mishev, I.G. Usoskin and G.A. Kovaltsov, Neutron monitor yield function: New improved computations, Journal of Geophysical Research: Space Physics 118 (2013) 2783.

[6] A.L. Mishev, S.A. Koldobskiy, G.A. Kovaltsov, A. Gil and I.G. Usoskin, Updated Neutron-Monitor Yield Function: Bridging Between In Situ and Ground-Based Cosmic Ray Measurements, Journal of Geophysical Research: Space Physics 125 (2020) e2019JA027433.

[7] S.A. Koldobskiy, G.A. Kovaltsov, A.L. Mishev and I.G. Usoskin, New Method of Assessment of the Integral Fluence of Solar Energetic (> 1 GV Rigidity) Particles from Neutron Monitor Data, Solar Physics 294 (2019) 94.

[8] M. Aguilar, L. Ali Cavasonza, B. Alpat, G. Ambrosi, L. Arruda, N. Attig et al., Observation of Fine Time Structures in the Cosmic Proton and Helium Fluxes with the Alpha Magnetic Spectrometer on the International Space Station, Physical Review Letters 121 (2018) 051101.

[9] S.A. Koldobskiy, G.A. Kovaltsov and I.G. Usoskin, Effective Rigidity of a Polar Neutron Monitor for Recording Ground-Level Enhancements, Solar Physics 293 (2018) 110.

[10] J.A. Van Allen, D.N. Baker, B.A. Randall and D.D. Sentman, The magnetosphere of Jupiter as observed with Pioneer 10: 1. Instrument and principal findings, Journal of Geophysical Research 79 (1974) 3559.

[11] S.A. Koldobskiy, V. Bindi, C. Corti, G.A. Kovaltsov and I.G. Usoskin, Validation of the neutron monitor yield function using data from AMS-02 experiment, 2011-2017, Journal of Geophysical Research: Space Physics 124 (2019) 2367 [1904 . 01929]. 
[12] A. Bruno, G.A. Bazilevskaya, M. Boezio, E.R. Christian, G.A. de Nolfo, M. Martucci et al., Solar Energetic Particle Events Observed by the PAMELA Mission, The Astrophysical Journal 862 (2018) 97 [1807 . 10183].

[13] I. Usoskin, S. Koldobskiy, G.A. Kovaltsov, A. Gil, I. Usoskina, T. Willamo et al., Revised GLE database: Fluences of solar energetic particles as measured by the neutron-monitor network since 1956, Astronomy \& Astrophysics 640 (2020) A17.

[14] O. Raukunen, M. Paassilta, R. Vainio, J.V. Rodriguez, T. Eronen, N. Crosby et al., Very high energy proton peak flux model, Journal of Space Weather and Space Climate 10 (2020) 24.

[15] S. Koldobskiy, O. Raukunen, R. Vainio, G.A. Kovaltsov and I. Usoskin, New reconstruction of event-integrated spectra (spectral fluences) for major solar energetic particle events, Astronomy \& Astrophysics 647 (2021) A132 [2101 . 10234].

[16] J.H. King, Solar proton fluences for 1977-1983 space missions, Journal of Spacecraft and Rockets 11 (1974) 401.

[17] J.N. Goswami, R.E. McGuire, R.C. Reedy, D. Lal and R. Jha, Solar flare protons and alpha particles during the last three solar cycles, Journal of Geophysical Research 93 (1988) 7195.

[18] J. Feynman, T.P. Armstrong, L. Dao-Gibner and S. Silverman, New interplanetary proton fluence model, Journal of Spacecraft and Rockets 27 (1990) 403.

[19] I. Jun, R.T. Swimm, A. Ruzmaikin, J. Feynman, A.J. Tylka and W.F. Dietrich, Statistics of solar energetic particle events: Fluences, durations, and time intervals, Advances in Space Research 40 (2007) 304.

[20] W.R. Webber, P.R. Higbie and K.G. McCracken, Production of the cosmogenic isotopes $3 H$, $7 \mathrm{Be}, 10 \mathrm{Be}$, and $36 \mathrm{Cl}$ in the Earth's atmosphere by solar and galactic cosmic rays, Journal of Geophysical Research: Space Physics 112 (2007) .

[21] I.G. Usoskin, S.A. Koldobskiy, G.A. Kovaltsov, E.V. Rozanov, T.V. Sukhodolov, A.L. Mishev et al., Revisited reference solar proton event of 23-Feb-1956: Assessment of the cosmogenic-isotope method sensitivity to extreme solar events, Journal of Geophysical Research: Space Physics 125 (2020) e2020JA027921 [2005 . 10597]. 